Pacific Journal of Mathematics

ON THE WALLMAN ORDER COMPACTIFICATION 


\title{
ON THE WALLMAN ORDER COMPACTIFICATION
}

\author{
D. C. KenT
}

\begin{abstract}
The Wallman order compactification $w_{0} X$ of a topological ordered space $X$ has been constructed by Choe and Park. This paper establishes necessary and sufficient conditions for their compactification to be $T_{2}$-ordered, in which case it coincides with the Nachbin (or Stone-Čech order) compactification.
\end{abstract}

Introduction. Let $(X, \leq)$ be a poset. For $x \in X$, let $i(x)=\{y \in X$ : $x \leq y\}$ and let $d(x)=\{y \in X: y \leq x\}$. If $A \subseteq X$, let $i(A)=\bigcup\{i(x)$ : $x \in A\}$, and $d(A)=\bigcup\{d(x): x \in A\}$. If $A=i A$ (respectively, $A=d(A)$ ), then $A$ is called an increasing (respectively, decreasing) set; a set which is either increasing or decreasing is said to be monotone.

A topological ordered space $(X, \leq, \tau)$ consists of a poset $(X, \leq)$ equipped with a topology $\tau$. If $\tau$ has an open subbase consisting of monotone sets, then the topological ordered space is said to be convex. Since only convex topological ordered spaces can have order compactifications which are $T_{2}$-ordered (see below), we shall henceforth consider only spaces of this type. For brevity, a convex topological ordered space $(X, \leq, \tau)$ will be simply called a space and designated by " $X$ ".

Following McCartan [4], we define a space $X$ to be $T_{1}$-ordered if $i(x)$ and $d(x)$ are both closed for all $x \in X$, and $T_{2}$-ordered if the partial order relation is a closed subset of $X \times X$. A $T_{1}$-ordered space is $T_{4}$-ordered (normally ordered in [5]) if, whenever $A$ and $B$ are closed disjoint subsets, the former decreasing and the latter increasing, there are disjoint open sets $U$ and $V$, the former decreasing and the latter increasing, such that $A \subseteq U$ and $B \subseteq V$. The " $T_{3}$-ordered" property is defined in [4], and " $T_{3.5}$-ordered" can be taken to mean "completely regular ordered" as defined in [5], but it will not be necessary to repeat these latter definitions here.

Nachbin has constructed a Stone-Čech type order compactification $\beta_{0} X$ of an arbitrary $T_{3.5}$-ordered space $X$ with the property that any continuous, increasing function from $X$ into a $T_{2}$-ordered, compact space can be lifted to $\beta_{0} X$. For details of the Nachbin compactification, see [3]. More recently, Choe and Park showed that $X$ is $T_{4}$-ordered whenever $w_{0} X$ is $T_{2}$-ordered, but were unable to prove the converse. Our main result establishes that $w_{0} X$ is $T_{2}$-ordered if and only if $X$ is strongly $T_{4}$-ordered 
(this term is defined below), and consequently that $w_{0} X$ and $\beta_{0} X$ are equivalent compactifications of a strongly $T_{4}$-ordered space $X$.

Let $X$ be a topological ordered spae. If $A \subseteq X$, let $I(A)$ (respectively, $D(A)$ ) be the smallest increasing (respectively, decreasing) closed set containing $A$, and let $\hat{A}=I(A) \cap D(A)$. Let $\mathscr{C}_{X}=\{A \subseteq X: A=\hat{A}\}$. Note that all members of $\mathscr{C}_{X}$ are closed and convex; we shall call the members of $\mathscr{C}_{X} c$-sets. All monotone closed sets are $c$-sets, and thus $\mathscr{C}_{X}$ is a closed subbase for $\tau$. One can easily verify that every set of the form $A^{\wedge}$, for $A \subseteq X$, is a $c$-set, and also that $\mathscr{C}_{X}$ is closed under finite intersections.

Let $F(X)$ be the set of all filters on $X$; the fixed ultrafilter generated by $\{x\}$ will be denoted by $\dot{x}$ for $x \in X$. If $\mathscr{F}, \mathscr{G} \in F(X)$, then $\mathscr{F} \vee \mathscr{G}$ will designate the filter generated by $\{F \cap G F \in \mathscr{G}, G \in \mathscr{G}\}$ (assuming that the latter collection does not include $\varnothing)$.

For $\mathscr{F} \in F(X)$, we denote by $i(\mathscr{F})$ the filter generated by $\{i(F)$ : $F \in \mathscr{G}\}$; the filters $d(\mathscr{F}), I(\mathscr{F})$, and $D(\mathscr{F})$ are defined analogously. A filter $\mathscr{F}$ is a $c$-filter (respectively, a convex filter) if it has a filter base of $c$-sets (respectively, convex sets). Note that $\mathscr{F}$ is a $c$-filter (respectively, a convex filter) iff $\mathscr{F}=I(\mathscr{F}) \vee D(\mathscr{F})($ respectively, $\mathscr{F}=i(\mathscr{F}) \vee d(\mathscr{F})$ ). A $c$-filter which is not property contained in any other $c$-filter will be called a maximal c-filter. A standard Zorn's Lemma argument establishes that every $c$-filter is contained in a maximal $c$-filter.

We can assume that $X$ is a $T_{1}$-ordered space and define $w_{0}(X)$ to be the set of all maximal $c$-filters on $X$. Note that the only convergent maximal $c$-filters are the fixed ultrafilters. It will be convenient to write $w_{0} X=\{\dot{x}: x \in X\} \cup X^{\prime}$, where $X^{\prime}$ is the set of all non-convergent maximal $c$-filters. An order relation " $\leqslant$ " for $w_{0} X$ is defined as follows: $\mathscr{F} \leq \mathscr{G}$ iff $I(\mathscr{F}) \subseteq \mathscr{G}$ and $D(\mathscr{G}) \subseteq \mathscr{F}$. It is a simple matter to verify that $\left(w_{0} X, \lesssim\right)$ is a poset and that the canonical map $\varphi:(X, \leq) \rightarrow\left(w_{0} X, \lesssim\right)$, defined by $\varphi(x)=\dot{x}$, is increasing.

We next introduce a topology on $w_{0} X$. For $A \subseteq X$, define $A^{*}=\{\mathscr{F} \in$ $\left.w_{0} X: A \in \mathscr{F}\right\}$. Then $\mathscr{C}^{*}=\left\{A^{*}: A \in \mathscr{C}_{X}\right\}$ is a closed subbase for a topology on $w_{0} X$ which we shall denote by $w_{0} \tau$. Clearly, $(A \cap B)^{*}=A^{*}$ $\cap B^{*}$ for all subsets $A, B$ of $X$; from this one easily deduces that $w_{0} X$ is a topological ordered space. It is obvious that $A=\varphi^{-1}\left(A^{*}\right)$ for any $A \subseteq X$; therefore $\varphi: X \rightarrow w_{0} X$ is a topological embedding, and both $\varphi$ and $\varphi^{-1} \mid \varphi(x)$ are increasing functions.

Before proceeding further, it is desirable to compare our construction of $w_{0} X$ with that of Choe and Park. They define a bifilter $(\mathscr{G}, \mathscr{H})$ on $X$ to be a pair of filters such that $\mathscr{G}$ has a base of decreasing closed sets, $\mathscr{H}$ has a base of increasing closed sets, and $\mathscr{G} \vee \mathscr{H}$ exists; the set of all maximal 
bifilters forms the underlying set for their compactification, which is also denoted by $w_{0} X$. It is easy to see that, for any bifilter $(\mathscr{G}, \mathscr{H})$ on $X$, the filter $\mathscr{F}=\mathscr{G} \vee \mathscr{H}$ is a $c$-filter, and that, for any $c$-filter $\mathscr{F},(D(\mathscr{F}), I(\mathscr{F}))$ is a corresponding bifilter. If $(\mathscr{G}, \mathscr{H})$ is a maximal bifilter, then $\mathscr{F}=\mathscr{G} \vee \mathscr{H}$ is a maximal $c$-filter, and $(D(\mathscr{F}), I(\mathscr{F}))=(\mathscr{G}, \mathscr{H})$; thus a bijection exists between the set of maximal bifilters on $X$ and the set of maximal $c$-filters on $X$. A comparison of the order relation and topology defined for $w_{0} X$ in [2] with our definitions given above reveals the equivalence of these spaces both as posets and as topological spaces. Thus the results obtained concerning $w_{0} X$ in [2] are applicable here, albeit with appropriate terminological alterations. The next two results are obtained in this way.

Proposition 1.1. For any $T_{1}$-ordered space $X,\left(w_{0} X, \varphi\right)$ is an order compactification of $X$, and $w_{0} X$ is a $T_{1}$ topological space. If $w_{0} X$ is $T_{2}$-ordered, then $X$ is $T_{4}$-ordered.

Proposition 1.2. Let $X$ be a $T_{1}$-ordered space, $Y$ a $T_{2}$-ordered compact space, and $f: X \rightarrow Y$ a continuous, increasing function. Then there is a unique, continuous, increasing function $\bar{f}: w_{0} X \rightarrow Y$ such that $\bar{f} \cdot \varphi=f$.

We define a $T_{4}$-ordered space $X$ to be strongly $T_{4}$-ordered if, whenever $A$ and $B$ are $c$-sets:

$$
\begin{array}{ccc}
I(A) \cap B=\varnothing & \text { implies } & I(A) \cap D(B)=\varnothing \\
D(A) \cap B=\varnothing & \text { implies } & D(A) \cap I(B)=\varnothing
\end{array}
$$

Note that a $T_{4}$-ordered space $X$ is strongly $T_{4}$-ordered iff, for a $c$-set $A$ and a decreasing open set $U$ with $A \subseteq U, D(A) \subseteq U$ and dually.

Priestly [6] defines a $C$-space to be a topological ordered space $X$ such that, for each closed subset $A, i(A)$ and $d(A)$ are also closed. The class of strongly $T_{4}$-ordered spaces includes the $T_{4} C$-spaces, among which are the $T_{2}$-ordered compact spaces.

Proposition 1.3. A $T_{1}$-ordered space $X$ is strongly $T_{4}$-ordered if and only $w_{0} X$ is $T_{2}$-ordered

Proof. In Proposition 1, page 26, [5], Nachbin shows that a space is $T_{2}$-ordered if, whenever $a \nless b$, there is an increasing neighborhood $V$ of a and a decreasing $W$ of $b$ such that $V \cap W=\varnothing$.

Assume that $\mathscr{F}, \mathscr{G}$ are elements of $w_{0} X$ such that $\mathscr{F} \leqq \mathscr{G}$ is false. Then either $I(\mathscr{F}) \subseteq \mathscr{F}$ or $D(\mathscr{G}) \subseteq \mathscr{F}$ is false. In the former case, since $\mathscr{G}$ is a 
maximal $c$-filter, there is $F \in \mathscr{F}$ and $G \in \mathscr{G}$ such that $I(F) \cap G=\varnothing$. By the assumption that $X$ is strongly $T_{4}$-ordered, $I(F) \cap D(G)=\varnothing$, and so there are disjoint open neighborhoods $U$ and $V$ of $I(F)$ and $D(G)$, respectively, such that $U$ is increasing and $V$ decreasing. Then $U^{*}$ and $V^{*}$ are disjoint, open neighborhoods of $\mathscr{F}$ and $\mathscr{G}$, respectively, in $w_{0} X$, the former increasing and the latter decreasing. This $w_{0} X$ is $T_{2}$-ordered.

Conversely, assume that $w_{0} X$ is $T_{2}$-ordered. Let $A, B$ be $c$-sets and suppose $I(A) \cap B=\varnothing$. Then $I(A)^{*} \cap B^{*}=\varnothing . I(A)^{*}$ is a closed, increasing subset of $w_{0} X$ and $B^{*}=D(B)^{*} \cap I(B)^{*}$ is a closed subset of $w_{0} X$. Let $d_{w}\left(B^{*}\right)=\left\{\mathscr{F} \in w_{0} X: \mathscr{F} \leq \mathscr{G}\right.$ for some $\left.\mathscr{G} \in B^{*}\right\}$. By Proposition 4, page 44, [5], $d_{w}\left(B^{*}\right)$ is a closed subset of $w_{0} X$, and it follows that $I(A)^{*} \cap d_{w}\left(B^{*}\right)=\varnothing$. Then $\varphi^{-1}\left(I(A)^{*} \cap d_{w}\left(B^{*}\right)\right)=\varphi^{-1}\left(I(A)^{*}\right) \cap$ $\varphi^{-1}\left(d_{w}\left(B^{*}\right)\right)=\varnothing$. Since $\varphi^{-1}\left(I(A)^{*}\right)=I(A)$ and $D(B) \subseteq \varphi^{-1}\left(d\left(B^{*}\right)\right)$, it follows that $I(A) \cap D(B)=\varnothing$. A similar argument shows that if $D(A)$ $\cap B=\varnothing$, then $D(A) \cap I(B)=\varnothing$. This conclusion that $X$ strongly $T_{4^{-}}$ ordered now follows with the help of Proposition 1.1

Corollary 1.4. $A T_{4}$-ordered space $X$ is strongly $T_{4}$-ordered if and only if, for any c-set $A, d(A)$ and $i(A)$ are both closed.

Proof. The condition is obviously sufficient. Suppose that $X$ is strongly $T_{4}$-ordered and $x \notin d(A)$. Then $i(x)^{*} \cap A^{*}=\varnothing$, and consequently $i(x)^{*}$ $\cap d_{w}\left(A^{*}\right)=\varnothing$. It follows that $i(x) \cap \varphi^{-1}\left(d_{w}\left(A^{*}\right)\right)=\varnothing$. Since the closure of $d(A)$ in $X$ is a subset of $\varphi^{-1}\left(d_{w}\left(A^{*}\right)\right), x$ is not in the closure of $d(A)$. Thus $d(A)$ is closed.

Corollary 1.5. Let $X$ be $T_{3.5}$-ordered. Then the compactifications $w_{0} X$ and $\beta_{0} X$ are equivalent if and only if $X$ is strongly $T_{4}$-ordered.

If the order relation of $X$ is trivial, then the $c$-sets are simply the closed sets, and the compactification $w_{0} X$ is identical with the ordinary Wallman compactification. In this case, Corollary 1.5 yields the wellknown equivalence of the Wallman and Stone-Čech compactifications for $T_{4}$ topological space.

We conclude by considering the Wallman order compactification for a simple and familiar class of spaces. We define a totally ordered space to be a totally ordered set with its order topology. If $X$ is a totally ordered space, then one can show that $w_{0} X$ (and hence $\beta_{0} X$ ) is a totally ordered space and a complete lattice. If $X=R$ is the totally ordered space of real numbers, then $w_{0} X$ can be identified with the extended real line $[-\infty, \infty]$. 
If $X=Q$ is the space of rationals, then $w_{0} X$ can also be regarded as the extended real line, but with each irrational "occurring twice"; by identifying these "irrational pairs", one obtains $w_{0} R$ as a quotient space of $w_{0} Q$.

\section{REFERENCES}

[1] T. H. Choe and Y. H. Hong, Exensions of completely regular ordered spaces, Pacific J. Math., 64 (1976), 37-48.

[2] T. H. Choe and Y. S. Park, Wallman's type order compactification, Pacific J. Math., 82 (1979), 339-347.

[3] g. Hommel, Increasing Radon measures on locally compact ordered spaces, Rendiconti di Matematica, 9 (1976), 85-117.

[4] S. D. McCartan, Separation axioms for topological ordered spaces, Proc. Comb. Phil. Soc., 64 (1968), 965-973.

[5] L. Nachbin, Topology and Order, Van Nostrand Mathematical Series 4, Princeton, N. J. 1965.

[6] H. A. Priestly, Ordered topological spaces and the representation of distributive lattices, Proc. London Math. Soc., (3) 24 (1972), 507-530.

Received October 14, 1983 and in revised form January 12, 1984.

WASHINGTON STATE UNIVERSITY

Pullman, WA 99164-2930 



\title{
PACIFIC JOURNAL OF MATHEMATICS EDITORS
}

DONALD BABBITT (Managing Editor)
University of California
Los Angeles, CA 90024
CHARLEs R. DEPrIMA
California Institute of Technology
Pasadena, CA 91125
R. FINN
Stanford University
Stanford, CA 94305

DoNAld BABBitT (Managing Editor)

Hermann FlaschKa

University of Arizona

Tucson, AZ 85721

RAmesh A. Gangolli

University of Washington

Seattle, WA 98195

ROBION KIRBY

University of California

Berkeley, CA 94720

C. C. MOORE

University of California

Berkeley, CA 94720

\author{
Hugo Rossi \\ University of Utah \\ Salt Lake City, UT 84112 \\ H. SAMELSON \\ Stanford University \\ Stanford, CA 94305 \\ HAROLD STARK \\ University of California, San Diego \\ La Jolla, CA 92093
}

ASSOCIATE EDITORS

R. ARENS

E. F. BECKENBACH
(1906-1982)

B. H. NEUMANN

F. WOLF

K. Yoshida

\section{SUPPORTING INSTITUTIONS}

UNIVERSITY OF ARIZONA

UNIVERSITY OF BRITISH COLUMBIA

CALIFORNIA INSTITUTE OF TECHNOLOGY

UNIVERSITY OF CALIFORNIA

MONTANA STATE UNIVERSITY

UNIVERSITY OF NEVADA, RENO

NEW MEXICO STATE UNIVERSITY

OREGON STATE UNIVERSITY
UNIVERSITY OF OREGON

UNIVERSITY OF SOUTHERN CALIFORNIA

STANFORD UNIVERSITY

UNIVERSITY OF HAWAII

UNIVERSITY OF TOKYO

UNIVERSITY OF UTAH

WASHINGTON STATE UNIVERSITY

UNIVERSITY OF WASHINGTON 


\section{Pacific Journal of Mathematics}

\section{Vol. 118, No. $1 \quad$ March, 1985}

Dan Amir, On Jung's constant and related constants in normed linear spaces ...1 Abdul Aziz, On the location of the zeros of certain composite polynomials . . 17 Joseph Barback, On hereditarily odd-even isols and a comparability of

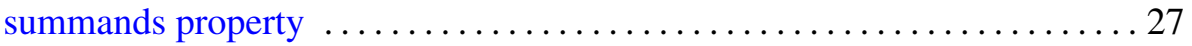

Matthew G. Brin, Klaus Johannson and Peter Scott, Totally peripheral 3-manifolds ........................................ 37

Robert F. Brown, A topological bound on the number of distinct zeros of an

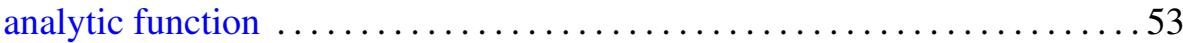

K. C. Chattopadhyay, Not every Lodato proximity is covered .......... 59

Beverly Diamond, Some properties of almost rimcompact spaces . .......63 63

Manfred Dugas and Rüdiger Göbel, On radicals and products . ......... 79

Abdelouahab El Kohen, A hyperbolic problem .................. 105

Harry Gonshor, Remarks on the Dedekind completion of a nonstandard model of the reals ................................... 117

William H. Kazez, On equivalences of branched coverings and their action

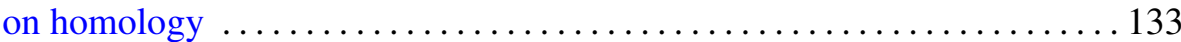

Darrell Conley Kent, On the Wallman order compactification .......... 159

Martin Andrew Magid, Lorentzian isoparametric hypersurfaces . . . . . . 165

Milan Miklavčič, Stability for semilinear parabolic equations with noninvertible linear operator

Richard Dean Neidinger and Haskell Paul Rosenthal, Norm-attainment of linear functionals on subspaces and characterizations of Tauberian operators

Johannes Vermeer, Closed subspaces of $H$-closed spaces 